Interventions

\title{
COVID-19 RNA Testing Technology Assessment
}

Fahim Farzadfard ${ }^{1}$, Louis Kang ${ }^{2}$, Samantha Bates ${ }^{3}$, Karthik Dinakar ${ }^{4}$, Samuel J. Klein ${ }^{5}$, Jack Kreindler, A. James Phillips ${ }^{6}$, Jess Sousa ${ }^{3}$, Amelia Wattenberger, James Weis, Joichi Ito ${ }^{7}$

${ }^{1}$ Senior Schmidt Science Fellow, ${ }^{2}$ Center of Complex Intervention, MitoLab,

${ }^{3}$ Center of Complex Interventions, ${ }^{4}$ Massachusetts Institute of Technology,

${ }^{5}$ Knowledge Futures Group, ${ }^{6}$ Open Cell Labs, ${ }^{7}$ Center of Complex Interventions, Keio University

Published on: Apr 23, 2020

DOI: 10.21428/9610ddb2.46de84ab

License: Creative Commons Attribution 4.0 International License (CC-BY 4.0). 


\title{
COVID-19 RNA Testing Technology Assessment
}

\section{Molecular diagnostic strategies for the COVID-19 pandemic}

Version 2.0

Fahim Farzadfard, Louis Kang, Samantha L. Bates, Karthik Dinakar, Jack Kreindler, Samuel Klein, A. James Phillips, Jessica Sousa, Amelia Wattenberger, James W. Weis, and Joichi Ito 1

\begin{abstract}
With the ongoing COVID-19 pandemic, and in response to high demand, a multitude of diagnostic tests for the detection and monitoring of the spread of the SARS-CoV-2 virus have been developed. This living document aims to survey the technical aspects of these diagnostic tests, outline the features and current limitations of their underlying technologies, formulate a modular framework for the assessment of the existing (and upcoming) tests workflows, and help depict a clearer and more comprehensive picture of this rapidly evolving space. With this manuscript as a starting point, we aim to help orchestrate a community effort to identify potential pitfalls and bottlenecks in the existing testing workflows---with the ultimate goal of paving the way to more sensitive, robust, scalable, and widespread tests for maximal social impact.
\end{abstract}

\section{This document}

- This document is a work in progress. The intention is to make it available publicly to solicit feedback and to be a living document. Please check back for the latest version at https://interventions.centerofci.org/pub/covid-testing-assessment/

- The document is Creative Commons Attribution licensed - share freely with attribution.

\section{Brief Background on SARS CoV-2}

- SARS-CoV-2 (or Severe Acute Respiratory Syndrome Coronavirus 2) causes COVID19 (also known as Coronavirus Disease 2019).

- SARS-CoV-2 first appeared in December 2019 in Wuhan, China and rapidly spread to over 160 countries, with over 2.4 million individuals infected and over 160,000

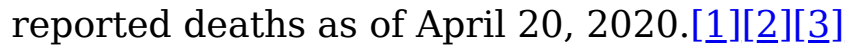

- The current evidence indicates that the majority of documented cases are the result of infection from undiagnosed individuals. [4] This is likely due, at least in part, to the 
high levels of viral shedding apparent in individuals without, or with minimal, symptoms.[ㅁ]

- There is currently no effective vaccine or widely accepted treatment for SARS-CoV-2. This, combined with the uncertainties around clinical diagnosis, the high viral shedding levels of minimally asymptomatic patients, and the high rate of infection from unknown sources makes the development of a widely-available and rapid diagnostic test with good performance characteristics (e.g. sensitivity and specificity) of critical concern. However, such tests have not yet been deployed in many countries in the world, including the United States.

- The efficacy of the rapid deployment of widespread testing to the most heavily infected areas, in combination with digital surveillance, strict quarantine, and large stockpiles of personal protective equipment, has been demonstrated through the remarkable first wave containment of SARS-CoV-2 in Singapore (which is experiencing a second wave of cases), Taiwan, South Korea, and Hong Kong.

- SARS-CoV-2 belongs to the broad family of viruses known as coronaviruses. Coronaviruses are positive-sense single-stranded RNA (+ssRNA) viruses.[] ]

- The SARS-CoV-2 RNA genome size is 29,891 nucleotides, encoding for 9,860 amino acids.

- As of 20 April 2020, over 4500 SARS-CoV-2 genomes sampled on six continents were publicly available.[]]

- The SARS-CoV-2 has 96\% nucleotide identity with BatCoV RaTG13,[8] 89\% with bat SARS-like-CoVZXC21 and CoVZC45, and 82\% with that of human SARS-CoV.

- SARS-CoV-2 consists of a lipid bilayer where the membrane, envelope, and spike structural proteins are anchored and encapsulate the virus RNA genome.

- Like other coronaviruses, SARS-CoV-2 has four structural proteins, known as the S (spike), E (envelope), M (membrane), and N (nucleocapsid) proteins

- The N protein holds the RNA genome

- The S, E, and M proteins together with the lipid bilayer create the viral envelope.

- The spike protein is the protein that enables the virus to attach to the host cell. 


\section{Current Situation}

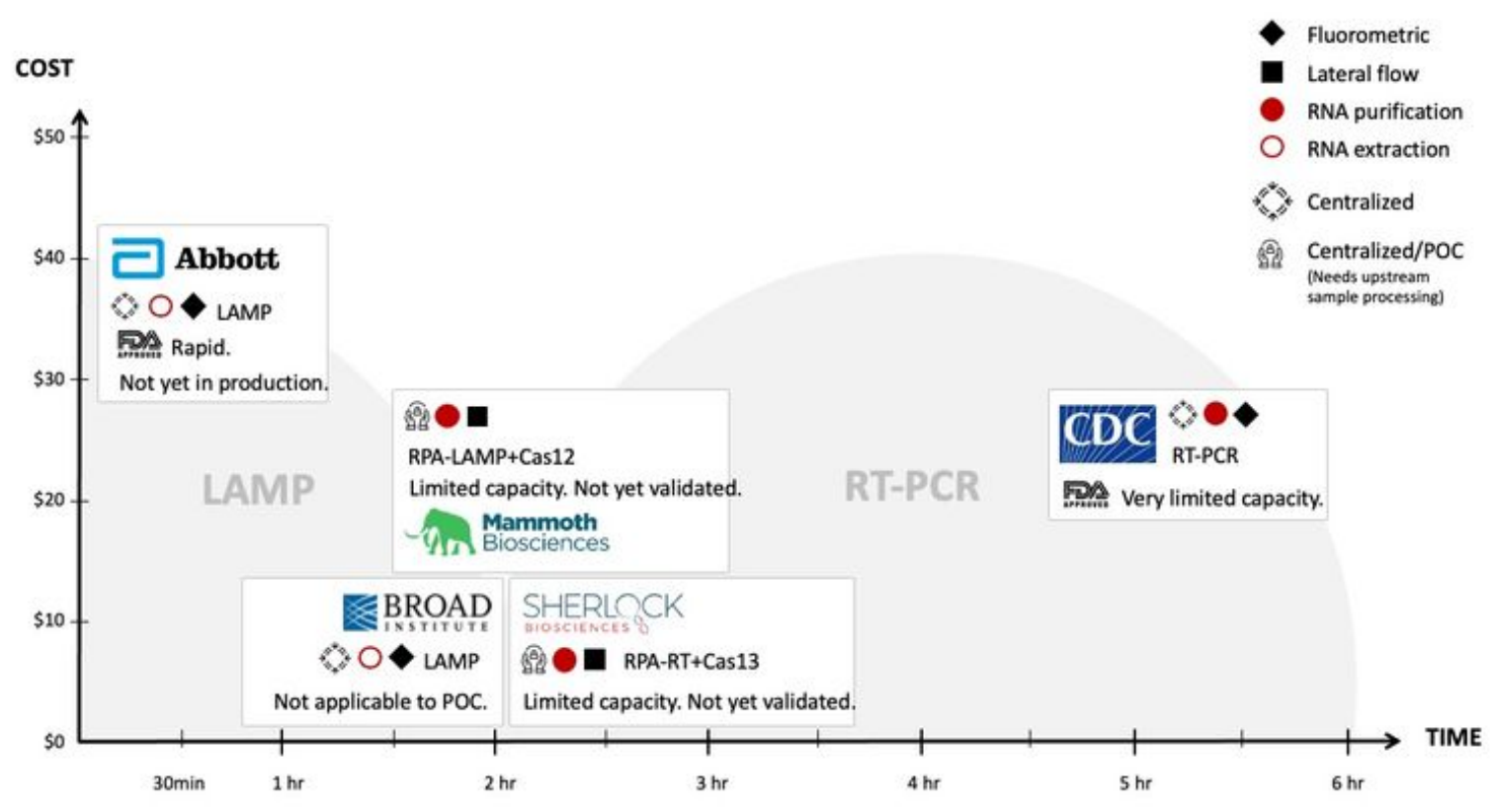

- Currently, in the United States, most CDC-certified SARS-CoV-2 real-time reverse transcription PCR (RT-qPCR) diagnostic tests have a laboratory turnaround time of approximately 4-6 hours, with results that can be delayed for $>24$ hours after sample collection due to shipping requirements, and are only slightly more than $50 \%$ sensitive if performed once (twice $=71 \%$ ).

- New innovations in this regime are being pursued - as impactful as decreasing the readout time to 5 minutes. However, these tests are only available in CDCdesignated public health laboratories certified to perform high-complexity testing.

- The RT-qPCR tests rely on lengthy and resource-intensive RNA purification and RTqPCR workflows. The shortage of proprietary reagents needed for these workflows has been a bottleneck in the diagnosis capacity of laboratories in the US and around the globe in the recent outbreak.

- Demand could rise to $50 \%$ of the US population, while the projected testing capacity based on current infrastructure and methods, over the next few months, is expected to be $\sim 100 \mathrm{~K}$ tests per day.

\section{Molecular Diagnostic for SARS-CoV-2}

Molecular diagnostics for SARS-COV-2 can be categorized into direct and indirect approaches: 
- Direct approaches assess the presence of viral components (RNA or proteins/antigens) in at-risk individuals and are suitable for identifying individuals who have contracted the virus.

- Indirect approaches detect the presence of molecules/agents (e.g. antibodies) produced by the human body in response to this specific virus. These approaches are suitable for identifying patients who have previously contracted the virus but where the current infection is not necessarily still present. Future antibody serology assays may also demonstrate a degree of immunity to further infection.

In this technical assessment, we will mainly focus on diagnostic approaches that rely on nucleic acids (RNA) detection since they are most relevant to direct testing. Protein detection could be a complementary approach to nucleic acid detection, however, it is less robust and sensitive and more prone to errors due to the complexities of working with proteins. Given the high suspected rate of minimally-symptomatic infections, indirect approaches may also be useful in providing a mechanism to quantify the prevalence of immunity in the general population.[ㅁ]

\section{SARS-CoV-2 Direct Molecular Diagnostics: general steps}

In general, the molecular diagnostics workflows can be described as the following steps: 1) sampling, 2) upstream sample processing, 3) detection and signal amplification, and 4 ) readout. In this section we discuss the advantages and limitations of the existing strategies for each of these steps:

\section{Sampling: to get usable samples from patients}

This is a manual step and common amongst all diagnostics methods. Given that Covid19 is an upper respiratory tract infection, nasal swabs have been the main form of sampling for direct (RNA or protein) detection while blood samples have been used for indirect (antibody) tests. $\underline{2}$ Tests based on alternative, less invasive sampling methods are also being developed, one of which (based on saliva samples) recently received Emergency Use Authorization.[1ㅜ]

\section{Upstream sample processing: to release (purify or extract) viral RNA from} other agents present in the sample to make them accessible to detection reagents.

Upstream sample processing such as RNA purification helps to achieve robust and sensitive detection and minimize possible false-negatives. Depending on the detection and the signal amplification strategy and readout used in a given workflow, the level of this dependency could be different. For example, a typical workflow based on $\underline{\text { RT-qPCR }}$ 
requires RNA purification to achieve sensitive detection as impurities in the samples could inhibit DNA polymerases. On the other hand, isothermal amplification, an alternative to PCR, involves methods that are less sensitive to sample impurities and minimal sample treatment (one-step RNA extraction) would be sufficient to detect and amplify the signal in most clinical samples. So, strategies that require no or minimal sample treatments are more scalable:

\section{RNA purification vs. RNA extraction}

- $\boldsymbol{R N A}$ purification is a lengthy process (takes more than 30 minutes) that involves multiple washes and purification steps. Column-based RNA purification and phenolchloroform extraction are the two most commonly used protocols for RNA purification. However, these strategies do not easily scale to millions of samples, as they are not compatible with high-throughput workflows and require manual processing. State-of-the-art RNA purification machines (e.g., from Qiagen) are relatively expensive and can only process a few dozen samples at a time. Furthermore, many of the commercially available reagents are proprietary which makes large-scale production of these reagents by third parties even more challenging. The shortage of RNA purification reagents is a bottleneck that limits the capacity of current detection kits that rely on RT-qPCR. While this shortage could be partially addressed by the ramp-up of their reagents, RNA purification remains a slow and laborious process. The FDA has issued Emergency Use Authorizations for many covid-19 tests,[11] but many of these EUA documents do not explicitly mention the RNA purification step.

- $\boldsymbol{R N A}$ extraction is the release of the RNA genome from viral particles and can be achieved by simply suspending the swab in a lysis buffer or heating the samples for a few minutes. While the process does not separate the RNA from other agents, it can release enough RNA that can be detected by a robust detection and signal amplification method (e.g., isothermal amplification) that is less sensitive to (and thus not inhibited by) impurities in the sample. RNA extraction is a simple, cheap, and time-saving alternative to RNA purification that can be easily scaled to tens of thousands of samples. When using RNA extraction, the sensitivity of detection can be compensated for by using more sensitive signal detection and amplification methods and readouts.[1ㅡㄹ $[\underline{13}][\underline{14}]$

RNA extraction and RNA purification have been used interchangeably in various contexts. As a rule of thumb, one can consider workflows that only involve the release of RNA moieties into the medium (e.g., via heat treatment or lysis buffer) as RNA 
extraction and those that involve additional RNA binding and wash steps as RNA purification.

\section{Visit the web version of this article to view interactive content.}

Comparison of RNA purification and extraction.

\section{Detection and signal amplification: to detect the presence of viral} components and produce/amplify signals (e.g., by RT-qPCR) so that they can be read out.

The nucleic acid detection and amplification methods that can be applied to the CoV-2 RNA genome can be categorized into three main classes: 1) RT-qPCR, 2) RNA Isothermal amplification, and 3) CRISPR-based methods. The first two methods involve reverse-transcription of segment(s) of the viral RNA in samples into cDNA (using a reverse-transcriptase enzyme) and amplifying the cDNA millions of times using PCR or isothermal amplification methods which can then be detected by a readout strategy. The CRISPR-based methods can be used to directly detect the viral RNA (using the Cas13 enzyme) or after converting the RNA into cDNA through reverse transcriptase (using the Cas12 enzyme). These methods have different degrees of reliance on specialized equipment, trained personnel, and upstream sample processing. The pros and cons of each of these strategies are described below:

- $\boldsymbol{R T - \boldsymbol { q P C R }}$ is the gold standard for molecular diagnostics of RNA viruses. It is a highly sensitive ${ }^{3}$ and well-established PCR-detection method that has been extensively used for the detection of various pathogens including CoV-2. Almost all the CoV-2 detection kits that are currently commercially available use RT-qPCR. However, for sensitive and robust detection, RT-qPCR requires an RNA purification step which makes the workflow laborious and slow that can only be performed by trained personnel. Without purified RNA the assay sensitivity drops significantly as DNA polymerases are inhibited by impurities in the samples, causing false-negatives; for example, human saliva contains RNA-degrading enzymes which leads to increased noise. The RT-qPCR workflow is slow and takes at least a few hours end-to-end. The time it takes to receive test results may also be longer if it is necessary to ship patient samples to locations that have the equipment and trained personnel to perform the test. Furthermore, the process requires relatively expensive qPCR equipment (the cheapest we could find on the market costs $\sim \$ 6 \mathrm{~K}$ ) which is 
significant for low volume - in addition to the reagents needed for each test While the process can be performed in a thermocycler machine that is relatively cheaper, the less expensive equipment may decrease sensitivity and lead to increased falsenegatives. Furthermore, there is evidence that the detection sensitivity for SARSCoV-2 may be particularly poor despite the attomolar limit of detection, with some studies showing only $47-59 \%$ of positive cases are identified via RT-qPCR. This reduction is suspected to be a consequence of loss, degradation, and/or mutation of the viral RNA, and addressing this concern has been a focus of recent research. [15] [16]

- Isothermal amplification methods such as Loop-Mediated Isothermal Amplification (LAMP) and Recombinase Polymerase Amplification ( successfully applied to detect various pathogens (RNA and DNA viruses and bacteria) in patient (blood, serum, saliva, etc.) and environmental (soil, water, etc.) samples, with minimal sample extraction or no sample treatments. The amplification is performed at a constant temperature (even room temperature), thus heavily reducing the reliance on specialized equipment and the cost. DNA amplification is very fast, and signals can be detected in as little as 10 minutes.

- CRISPR-based methods are the newest player in molecular diagnostics and their robustness and performance need to be tested with clinical samples. As opposed to the above-mentioned methods, which rely on RNA/DNA amplification, CRISPR-based methods rely on the capacity of CRISPR effector proteins (Cas12 and Cas13) to discriminate the desired sequence with single base-pair resolution. The collateral nuclease activity of these proteins at the presence of their DNA or RNA target is used to degrade a nucleotide probe and create a colorimetric or fluorogenic signal. Similar to RT-qPCR, CRISPR-based methods also require an RNA purification step for robust and sensitive detection and signal amplification. A pre-amplification step using an isothermal amplification method could help to increase the sensitivity of these methods, however, as a result of such reliance, the success of CRISPR-based methods would become dependent on the success of the initial amplification step.

Visit the web version of this article to view interactive content.

Comparison of amplification methods. Proprietary solutions: [a]

TwistDX

[b] UC Berkeley/Mammoth Biosciences [c] Broad Institute/Sherlock Biosciences 
4. Readout: to convert the amplified signal into a visualizable (calorigenic) or machine-readable (fluorescent, DNA sequence) form that can be quantified or qualified.

An amplified signal using one of the above-mentioned methods needs to be converted into a format that can be readout visually or by a machine.

Colorimetric and fluorometric readouts: In the case of DNA amplification methods (PCR and isothermal amplification), this can be achieved by adding colorigenic or fluorogenic DNA intercalating dyes that become visible or fluorescent upon binding to double-stranded DNA that is produced during DNA amplification, or by using fluorescent oligo probes that specifically interact with amplified DNA or are incorporated into it during amplification. Similar readouts can be achieved in CRISPRbased methods, using probes that become fluorescent or visible upon degradation by the collateral nuclease activity of activated Cas12 or Cas13. Fluorogenic substrates enable more sensitive and quantifiable readouts, however, they require optical equipment.

Lateral flow assays: Detection assays can be designed so that the nature of amplified DNA or oligo probes can be assessed by lateral flow (paper-based sample separation). This method provides a more sensitive and quantitative readout than bulk colorimetric assays; however, the sensitivity (signal-to-noise ratio) for this assay is less than for fluorometric assays. This lower sensitivity necessitates an RNA purification workflow to achieve robust detection and reduce false-negatives.

DNA sequence readout: In cases of RT-PCR and isothermal amplification methods, the amplified DNA can be directly sequenced. Adding DNA barcodes into primers enables us to uniquely mark each sample. Barcoded samples can be pooled together and analyzed on the same run by high-throughput sequencing methods, significantly reducing the cost per sample and improving the throughput of detection. However, this mode of detection requires access to Next Generation Sequencing (NGS) machines and is only applicable to centralized labs.

\section{Visit the web version of this article to view interactive} content.

Comparison of readout methods.

Visit the web version of this article to view interactive content. 


\section{Possible solutions to testing challenges}

- Alternative assays with shorter workflows: Even with the ramp-up of reagent production lines and the increase in the number of certified centers, certified RTqPCR workflows which involve lengthy and multi-step RNA purification and DNA amplification processes cannot be scaled to meet increasing demand. Thus, alternative and more easily scalable detection strategies are needed. RNA purification can be replaced by quick RNA extraction protocols which can be combined with sensitive, cheap, and robust detection methods, such as isothermal amplification, that are compatible with high-throughput assays. In this case, to compensate for the drop in the sensitivity of assays due to the use of RNA extraction (as opposed to RNA purification) in the workflow and minimize false-negatives, more sensitive readouts such as fluorometric assays could be used.

- Increasing the testing capacity of the centralized laboratories: It is clear that the testing demand is significantly higher than the current capacity of testing centers, and additional specialized testing centers are urgently needed. While valuable assets, services provided by centralized labs could be delayed due to shipment requirements. Furthermore, continuous monitoring of at-risk populations especially at remote locations may not be feasible as that would require routine (e.g., weekly) sampling and shipments for each at-risk individual. Therefore, employing more efficient testing methods at testing centers and developing more point-of-care diagnostic tools could be the best way to increase our testing capacity.

- Point of care (POC) diagnostics: The rapid outbreak of the COVID-19 pandemic across the globe highlights the need for point-of-care (POC) diagnostic tools that enable fast, robust, sensitive, and wide-spread detection and monitoring of emerging pathogens to achieve an effective public health response. Complementary to centralized labs, low-cost, easy-to-operate POC devices can serve as effective tools to monitor and combat the spread of the disease. Furthermore, these devices can be designed to immediately transmit test results and substantial relevant metadata (location, time, environmental conditions) to secure databases for further analysis, providing healthcare officials and decision-makers with valuable information, such as the extent and dynamics of viral spread in exposed populations, and of pandemic response.

Visit the web version of this article to view interactive content. 


\section{Case studies $\underline{4}$}

\section{Visit the web version of this article to view interactive content. \\ Comparison of a range of FDA-approved RNA tests \\ * Cost per test. Excludes instrument cost. \\ ** Up to 1 day including sample shipment.}

The sensitivity and robustness of the assay is a function of the methods used for each of the above mentioned four steps. Ideally, we want to:

1. Maximize assay sensitivity and robustness to reduce possible false negatives

While at the same time optimizing the following steps to the extent that doing so does not compromise the assay and lead to unwanted false negatives (producing negative results for a patient that is indeed positive): $\underline{5}$

2. Reduce workflow cycle: increase throughput and save time

3. Minimize cost

4. Increase wide-spread accessibility (no reliance on supply chain and shipment infrastructure, easy-to-operate workflow without training requirement)

Considering these criteria and the details discussed for methods available for each of the four steps, a testing technology addressing specific needs can be evaluated and designed. For example, an ideal workflow for a distributable, point-of-care testing method would be something like this:

1. Sampling

2. RNA extraction (as opposed to the lengthy RNA purification used in the existing workflows): cheap and quick workflow

3. Isothermal amplification (LAMP): robust and sensitive, isothermal (no requirement for specialized equipment), cheap and non-proprietary reagents and technique, less sensitive to inhibitory agents in unpurified samples.

4. Fluorometric detection: sensitive, real-time monitoring. High signal to noise ratio, which could compensate for a drop in sensitivity as a result of using RNA extraction instead of purification in the workflow. 
Visit the web version of this article to view interactive content.

\section{Additional Resources}

Testing and Lists of Tests

- Cheng, M. P., Papenburg, J., Desjardins, M., Kanjilal, S., Quach, C., Libman, M., Dittrich, S., \& Yansouni, C. P. (2020). Diagnostic Testing for Severe Acute Respiratory Syndrome-Related Coronavirus-2: A Narrative Review. Annals of Internal Medicine. https://doi.org/10.7326/M20-1301

- Coronavirus Test Tracker: Commercially Available COVID-19 Diagnostic Tests. (n.d.). 360Dx. Retrieved April 15, 2020, from https://www.360dx.com/coronavirus-testtracker-launched-covid-19-tests

- Sheridan, C. (2020). Fast, portable tests come online to curb coronavirus pandemic. Nature Biotechnology. https://doi.org/10.1038/d41587-020-00010-2

- COVID testing UPDATE. (n.d.). Open Cell. Retrieved April 15, 2020, from https://www.opencell.bio/news/covid-testing-update

- Hodgson, J. (2020). The pandemic pipeline. Nature Biotechnology. https://doi.org/10.1038/d41587-020-00005-z

- Health, C. for D. and R. (2020). Emergency Use Authorizations. FDA. https://www.fda.gov/medical-devices/emergency-situations-medicaldevices/emergency-use-authorizations

- Testing | Coronavirus Tech Handbook. (n.d.). Retrieved April 15, 2020, from https://coronavirustechhandbook.com/testing

Dashboards, General Information and Lists

- Science papers you should be reading about the coronavirus. (2020, March 31). Fred Hutch. https://www.fredhutch.org/en/news/center-news/2020/03/coronavirus-latestscientific-research.html

- CORD-19 dataset | Semantic Scholar. Retrieved April 19, 2020, from https://pages.semanticscholar.org/coronavirus-research

- COVID-19 Primer. (n.d.). Retrieved April 15, 2020, from https://covid19primer.com/dashboard 


\section{Footnotes}

1. This document was originally created by Farzadfard and Kang of MitoLab as a supporting document for Ito and the Center of Complex Interventions in the context of fundraising for the development of a COVID-19 test kit in collaboration with Conservation X Labs. Numerous edits and comments were contributed during a prepublication review phase. We will continue to version this document as additional information becomes available and more feedback is received. Please contribute to this document via the comment feature and check back for updates. $\triangleq$

2. While shortage of sampling supplies and swabs have been reported on some occasions, with the ramp up of production, the shortage has been resolved and doesn't seem to be an issue around these supplies anymore. $ヒ$

3. Sensitivity is subject to various additional factors, such as the extraction process and sample quality. $\subseteq$

4. These are some of the better-known tests in various direct testing categories, but is by no means exhaustive. For more complete lists, please see some of the links in the addition resources section. $\triangleq$

5. A recent case illustrates how compromising assay sensitivity and robustness could lead to defective tests and cause big problems: "Countries Rush to Dump 'Defective' Chinese COVID-19 Tests" and "S $\underline{\text { Chinese coronavirus test kits" }} \triangleq$

\section{Citations}

1. Zhu, N., Zhang, D., Wang, W., Li, X., Yang, B., Song, J., ... Tan, W. (2020). A Novel Coronavirus from Patients with Pneumonia in China, 2019. New England Journal of Medicine, 382(8), 727-733. https://doi.org/10.1056/nejmoa2001017

2. CDC. (2020, April 14). International Locations with Confirmed COVID-19 Cases. Centers for Disease Control and Prevention. https://www.cdc.gov/coronavirus/2019$\underline{\text { ncov/cases-updates/world-map.html }} \subseteq$

3. Coronavirus Update (Live): 2,428,276 Cases and 166,126 Deaths from COVID-19 Virus Pandemic - Worldometer. (n.d.). Retrieved April 20, 2020, from https://www.worldometers.info/coronavirus/ $\leftrightarrows$ 
4. Li, R., Pei, S., Chen, B., Song, Y., Zhang, T., Yang, W., \& Shaman, J. (2020). Substantial undocumented infection facilitates the rapid dissemination of novel coronavirus (SARS-CoV-2). Science, 368(6490), 489-493.

https://doi.org/10.1126/science.abb3221

5. Zou, L., Ruan, F., Huang, M., Liang, L., Huang, H., Hong, Z., ... Wu, J. (2020). SARS-CoV-2 Viral Load in Upper Respiratory Specimens of Infected Patients. New England Journal of Medicine, 382(12), 1177-1179.

https://doi.org/10.1056/nejmc2001737

6. Weiss, S. R., \& Navas-Martin, S. (2005). Coronavirus Pathogenesis and the Emerging Pathogen Severe Acute Respiratory Syndrome Coronavirus. Microbiology and Molecular Biology Reviews, 69(4), 635-664.

https://doi.org/10.1128/mmbr.69.4.635-664.2005

7. Genomic epidemiology of novel coronavirus - Global subsampling. Nextstrain project. https://nextstrain.org/ncov/global $\leftrightarrows$ 8. Jaimes, J. A., André, N. M., Chappie, J. S., Millet, J. K., \& Whittaker, G. R. (2020). Phylogenetic Analysis and Structural Modeling of SARS-CoV-2 Spike Protein Reveals an Evolutionary Distinct and Proteolytically Sensitive Activation Loop. Journal of Molecular Biology, 432(10), 3309-3325. https://doi.org/10.1016/j.jmb.2020.04.009 9. Feuer, B. L. J., William. (2020, April 17). WHO warning: No evidence that antibody tests can show coronavirus immunity. CNBC. https://www.cnbc.com/2020/04/17/whoissues-warning-on-coronavirus-testing-theres-no-evidence-antibody-tests-show$\underline{\text { immunity.html }}=$

10. First Saliva Test for COVID-19 Approved for Emergency Use by FDA. (n.d.). The Scientist Magazine ${ }^{\circledR}$. Retrieved from https://www.the-scientist.com/newsopinion/first-saliva-test-for-covid-19-approved-for-emergency-use-by-fda-67416 11. Emergency Use Authorization, FDA https://www.fda.gov/medicaldevices/emergency-situations-medical-devices/emergency-use-authorizations 12. Schmid-Burgk, J. L., Li, D., Feldman, D., Słabicki, M., Borrajo, J., Strecker, J., ... Zhang, F. (2020). LAMP-Seq: Population-Scale COVID-19 Diagnostics Using a Compressed Barcode Space. BioRxiv, 2020.04.06.025635. https://doi.org/10.1101/2020.04.06.025635 
13. Ladha, A., Joung, J., Abudayyeh, O. O., Gootenberg, J. S., \& Zhang, F. (n.d.). A 5min RNA preparation method for COVID-19 detection with RT-qPCR, 6.

14. Formsgaard, A. S., \& Rosenstierne, M. W. (n.d.). An alternative workflow for molecular detection of SARS-CoV-2 - escape from the NA extraction kit-shortage Itextbar medRxiv. Retrieved from https://www.medrxiv.org/content/10.1101/2020.03.27.20044495v1

15. Li, C., Debruyne, D. N., Spencer, J., Kapoor, V., Liu, L. Y., Zhou, B., ... Liu, Z. (2020, March 14). Highly sensitive and full-genome interrogation of SARS-CoV-2 using multiplexed PCR enrichment followed by next-generation sequencing. Cold Spring Harbor Laboratory. https://doi.org/10.1101/2020.03.12.988246

16. Suo, T., Liu, X., Feng, J., Guo, M., Hu, W., Guo, D., ... Chen, Y. (2020, March 6). ddPCR: a more sensitive and accurate tool for SARS-CoV-2 detection in low viral load specimens. Cold Spring Harbor Laboratory. https://doi.org/10.1101/2020.02.29.20029439 $\triangleq$ 